\title{
Numerical Technique for a System of Nonlinear Partial Differential Equations of the Time-Fractional Order
}

\section{Kawala AM*}

Department of Mathematics, Faculty of Science, Helwan University, Cairo, Egypt

\begin{abstract}
In this article, generalized differential transform method (GDTM) has been employed to obtain traveling wave solutions for some Systems of nonlinear wave equations of time-fractional order. This demonstrates the validity and great potential of the generalized differential transform method (GDTM) for solving system of nonlinear fractional partial differential equations NFDEs. Thus NFDEs can be easily solved with less computational work for any analytic nonlinearity due to the properties and available algorithms of the generalized differential transform method (GDTM). The fractional derivative is described in the Caputo fractional derivative sense. The solutions are obtained in the form of rapidly convergent infinite series with easily computable terms.
\end{abstract}

Keywords: Fractional nonlinear wave equations systems; Caputo fractional derivative; Generalized differential transform method; Taylor series method

\section{Introduction}

Generalized differential transform technique is introduced to solve nonlinear wave equations systems of time-fractional order. The concept of differential transform (one-dimension) was first proposed and applied to solve linear and nonlinear initial value problems in electric circuit analysis by Zhou [1]. Ayaz developed differential transform method to two-dimensional problem for PDE's initial value problems [2-4]. Kurnaz et al. generalized differential transform method to $\mathrm{n}$-dimensional case in order to solve PDEs [5].

Using Generalized (two-dimension) differential transformation technique GDTM, a closed form series solution or an approximate solution can be obtained [6]. The differential transform method obtains an analytical solution in the form of a polynomial [7-9]. The fractional derivatives are described in the Caputo fractional derivative sense. It is distinct from the traditional high order Taylor's series method, which demands symbolic competition of the necessary derivatives of the data functions [10-12]. The Taylor series method is computationally taken long time for large orders. With this method, it is possible to obtain highly accurate results or exact solutions for differential equations. This paper investigates the applicability, effectiveness and accuracy of generalized differential transform method on nonlinear wave equations systems of timefractional order [13-16].

\section{Fractional Calculus}

There are various different definitions of the concept of a fractional derivative [17-19]. Some of these are Riemann-Liouville, GrunwaldLetnikow, Caputo, and generalized functions approach. The most generally used definitions are the Riemann-Liouville and Caputo derivatives [12].

\section{Definition 1}

A real $f(x), x>0$, is said to be in the space $C_{\mu} \mu \in R$, if there exists a real number $p(>\mu)$ such that $f(x)=x^{p} f_{1}(x)$, where $f_{1}(x) \in C[0, \infty)$, and it is said to be in the space $C_{\mu}{ }^{m}$ iff $f^{m} \in C_{\mu}, m \in N$.

\section{Definition 2}

The Riemann-Liouville fractional integral operator of order $\alpha \geq 0$ of a function

$$
\begin{aligned}
& f \in C_{\mu^{\prime}} \mu \geq-1, \text { is defined as } \\
& \mathcal{J}_{0}^{v} f(x)=\frac{1}{\Gamma(v)} \int_{0}^{x}(x-t)^{v-1} f(t) d t, \mathrm{v}>0 \\
& J^{0} f(x)=f(x) .
\end{aligned}
$$

It has the following properties. For $f \in C_{\mu^{\prime}} \mu \geq-1, \alpha, \beta \geq 0$, and $\gamma>1$ :

1. $J^{\alpha} J^{\beta} f(x)=J^{\alpha+\beta} f(x)$,

2. $J^{\alpha} J^{\beta} f(x)=J^{\beta} J^{\alpha} f(x)$,

$$
\mathcal{J}^{0} x^{\gamma}=\frac{\Gamma(\gamma+1)}{\Gamma(\alpha+\gamma+1)} \mathrm{x}^{\alpha+\gamma}
$$

Caputo introduced an alternative definition which has the advantage of defining integer order initial conditions for fractional order differential equations. The Caputo fractional derivative is considered here because it allows traditional initial conditions to be included in the formulation of the problem [13].

\section{Definition 3}

The fractional derivative of $f(x)$ in the Caputo sense is defined as

$$
\mathrm{D}_{*}^{v} f(x)=\mathcal{J}_{a}^{m-v} \mathrm{D}^{M} f(x)=\frac{1}{\Gamma(m-v)} \int_{0}^{x}(x-t)^{m-v-1} f^{(\mathrm{m})}(t) d t,
$$

For $m-1<v<m, m \in N, x>0, f \in C_{-}{ }^{m}$.

\section{Lemma}

1 if $m-1<\alpha<m, m \in N$, and $f \in C_{\mu}{ }^{m}, \mu \geq-1$, then

$$
\begin{aligned}
& D_{*}^{\alpha} J^{\alpha} f(x)=f(x), \\
& \mathcal{J}^{\alpha} \mathrm{D}_{*}^{v} f(x)-\sum_{k=0}^{m-1} f^{k}\left(0^{+}\right) \frac{x^{k}}{k !}, \quad x>0
\end{aligned}
$$

*Corresponding author: Kawala AM, Department of Mathematics, Faculty of Science, Helwan University, Cairo, Egypt ,Tel: +20 2 25569061; E-mail: Kawala_26_1@yahoo.com

Received April 13, 2018; Accepted May 16, 2018; Published June 10, 2018

Citation: Kawala AM (2018) Numerical Technique for a System of Nonlinear Partial Differential Equations of the Time-Fractional Order. J Appl Computat Math 7: 398. doi: 10.4172/2168-9679.1000398

Copyright: @ 2018 Kawala AM. This is an open-access article distributed under the terms of the Creative Commons Attribution License, which permits unrestricted use, distribution, and reproduction in any medium, provided the original author and source are credited. 
Citation: Kawala AM (2018) Numerical Technique for a System of Nonlinear Partial Differential Equations of the Time-Fractional Order. J Appl Computat Math 7: 398. doi: 10.4172/2168-9679.1000398

Page 2 of 6

\section{GDTM Generalized Differential Transform}

We shall derive the generalized DTM that we have developed for the numerical solution of nonlinear partial differential equations with space and time-fractional derivatives.

Consider a function of two variables $u(x, y)$ and suppose that it can be represented as a product of two single-variable functions, i.e., $u(x$, $y)=f(x) g(y)$. Based on the properties of the generalized differential transform, the function $\mathrm{u}(\mathrm{x}, \mathrm{y})$ can be represented as

$$
\begin{aligned}
& \mathrm{u}(x, y)=\sum_{k=0} F_{\alpha}(k)\left(x-x_{0}\right)^{k \alpha} \sum_{h=0} G_{\beta}(h)\left(y-y_{0}\right)^{h \beta} \\
& =\sum_{k=0}^{\infty} \sum_{h=0} U_{\alpha \beta}(k, h)\left(x-x_{0}\right)^{k \alpha}\left(y-y_{0}\right)^{h \beta} .
\end{aligned}
$$

Where $0<\alpha, \beta \leq 1, U_{\alpha \beta}(k, h)=F_{\alpha}(k) G_{\beta}(h)$ is called the spectrum of $u(x, y)$. The generalized two dimensional differential transform of the function $u(x, y)$ is given by

$$
U_{\alpha, \beta}(k, h)=\frac{1}{\Gamma(\alpha k+1) \Gamma(\beta h+1)}\left[\left(D_{*_{\mathrm{x} 0}}^{\alpha}\right)^{k}\left(D_{*_{y} 0}^{\beta}\right)^{h} u(x, y)\right]_{(x 0, y 0)}
$$

Where $\left(D_{x 0}^{\alpha}\right)^{k=} D_{x 0}^{\alpha} D_{x 0}^{\alpha} \ldots D_{x 0}^{\alpha}, k$-times. In case of $\alpha=1$ and $\beta=1$.

The Generalized differential transform GDTM (1) reduces to the classical differential transform DTM. Next we give some useful theorems about writing the generalized differential transform in equivalent forms under certain conditions [12].

\section{Theorem 1}

Suppose that $U_{\alpha, \beta}(k, h), V_{\alpha, \beta}(k, h)$, and $W_{\alpha, \beta}(k, h)$ are the differential transformations of the functions $u(x, y), v(x, y)$ and $w(x, y)$ respectively, then [14]

1. If $u(x, y)=v(x, y) \pm w(x, y)$ then $U_{\alpha, \beta}(k, h)=V_{\alpha, \beta}(k, h) \pm W_{\alpha, \beta}(k$, h)

2. If $u(x, y)=a v(x, y), a \in R$, then $U_{\alpha, \beta}(k, h)=a V_{\alpha, \beta}(k, h)$

3. If $u(x, y)=v(x, y) w(x, y)$ then $U_{\alpha, \beta}(k, h)=\sum_{r=0}^{k} \sum_{s=0}^{h} V_{\alpha, \beta}(r, h-s)$ $W_{\alpha, \beta}(k-r, s)$

4. If $u(x, y)=\left(x-x_{0}\right)^{n \alpha}\left(y-y_{0}\right)^{m \beta}$, then $U_{\alpha, \beta}(k, h)=\delta(k-n) \delta(h-m)$.

\section{Theorem 2}

If $u(x, y)=D_{x 0}^{\alpha} v(x, y), 0<\alpha \leq 1$, then the generalized differential transform (1) can be written as [14]

$$
U_{\alpha, \chi}(k, h)=\frac{\Gamma(\alpha(k+1)+1)}{\Gamma(\alpha k+1)} \mathbf{V}_{\alpha, \beta}(k+1, h) \cdot
$$

\section{Theorem 3}

Assume that $u(x, y)=f(x) g(y)$ and the function $f(x)=x^{\lambda} h(x)$, where $\lambda>-1$ and

$\mathrm{h}(\mathrm{x})$ has the generalized Taylor series expansion $h(\mathrm{x})=\sum_{n=0}^{\infty} a_{n}\left(x-x_{0}\right)^{\alpha k}$, If

(a) $\beta<\lambda+1$ and $\alpha$ is arbitrary or

(b) $\beta \geq \lambda+1$ and $\alpha$ is arbitrary and $a_{n=} 0$ for $\mathrm{n}=0,1, \ldots, \mathrm{m}-1$, where $m-1<\beta \leq m$ Then the generalized differential transform (1) becomes

$$
U_{\alpha, \beta}(k, h)=\frac{1}{\Gamma(\alpha k+1) \Gamma(\beta h+1)}\left[\left(D_{*_{x} 0}^{\alpha}\right)^{k}\left(D_{*_{y} 0}^{\beta}\right)^{h} u(x, y)\right]_{(x 0, y 0)}
$$

The details of some properties $[14,15]$.

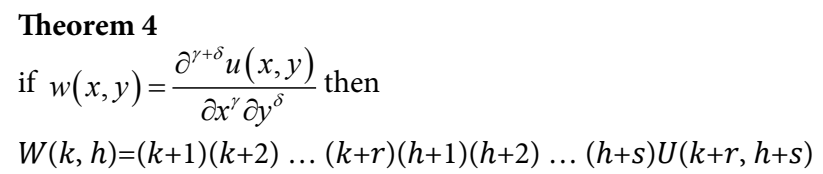

\section{Theorem 5}

If $w(x, y)=u(x, y) v(x, y)$, then $w(k, h)=\sum_{\gamma=0}^{k} \sum_{\delta=0}^{h} U(\gamma, h-s) V(k-\gamma, s)$.

\section{Theorem 6}

If $w(x, y)=x^{m} y^{n}$ then $W(k, h)=\delta(k-m, h-n)=\delta(k-m) \delta(h-n)$ where

$$
W(k, h)=\sum_{\gamma=0}^{k} \sum_{\delta=0}^{h} U(\gamma, h-s) V(k-\gamma, s)
$$

\section{Theorem 7}

$$
\begin{aligned}
& \text { If } w(x, y)=\frac{\partial u(x, y)}{\partial x} \frac{\partial v(x, y)}{\partial x} \text { then } \\
& W(k, h)=\sum_{\gamma=0}^{k} \sum_{S=0}^{h}(\gamma+1)(k-\gamma+1) U(\gamma+1, \mathrm{~h}-\mathrm{S}) V(k-\gamma+1, s)
\end{aligned}
$$

The proofs of Theorems are available in ref. [2].

\section{Applications}

In this section we consider examples that illustrate the performance and efficiency of the generalized differential transform method GDTM for solving nonlinear wave equations systems of time fraction order. Various numerical results are presented in the format of tables and graphs to make a comparison with results obtained by analytical.

Example 1. Consider the nonlinear KdV system of time-fractional order of the form [16]

$$
\begin{aligned}
& D_{t}^{{ }^{q}} u=-\alpha u_{x x x-} 6 \alpha u u_{x+} 6 v v_{x} \\
& D_{t}^{{ }^{q}} v=-\alpha v_{x x x-} 3 \alpha u v_{x}
\end{aligned}
$$

For $0<q<1$ subject to the initial conditions

$$
u(x, 0)=\beta^{2} \operatorname{Sech}^{2}\left(\frac{\gamma}{2}+\frac{\beta x}{2}\right), v(x, 0)-\sqrt{\frac{\alpha}{2}} \beta^{2} \operatorname{Sech}^{2}\left(\frac{\gamma}{2}+\frac{\beta x}{2}\right)
$$

The exact solitary wave solutions of the KdV system (2), for the special case $\mathrm{q}=1$ is given by

$$
\begin{aligned}
& u(x, t)=\beta^{2} \operatorname{sech}^{2}\left(\frac{1}{2}\left[\gamma-\alpha \beta^{3} t+\beta x\right]\right), \\
& v(x, t)=\sqrt{\frac{\alpha}{2}} \beta^{2} \operatorname{Sech}^{2}\left(\frac{1}{2}\left[\gamma-\alpha \beta^{3} t+\beta x\right]\right)
\end{aligned}
$$

where the constant $\alpha$ is a wave velocity and $\beta, \gamma$ are arbitrary constants.

Suppose that the solutions $\mathrm{u}(\mathrm{x}, \mathrm{t})$ and $\mathrm{v}(\mathrm{x}, \mathrm{t})$ can be represented as the products of single-valued functions, respectively.

Applying the generalized differential transform GDTM to both sides of (2), and by using the related theorems, we will have

$$
\begin{aligned}
U[i, j+1] & =\frac{\Gamma[q j+1]}{\Gamma[q(\mathrm{j}+1)+1]}(-\alpha(i+1)(i+2)(i+3)) \mathrm{U}[i+3, \mathrm{j}] \\
& -6 \alpha \sum_{\gamma=0}^{i} \sum_{s=0}^{j}((i-\gamma+1)) U[\gamma, \mathrm{j}-\mathrm{s}] U[i-\gamma+1, \mathrm{~s}] \\
& +6 \sum_{\gamma=0}^{i} \sum_{s=0}^{j}((i-\lambda+1)) V[\gamma, j-s] V[i-\gamma+1, s]
\end{aligned}
$$


Citation: Kawala AM (2018) Numerical Technique for a System of Nonlinear Partial Differential Equations of the Time-Fractional Order. J Appl Computat Math 7: 398. doi: 10.4172/2168-9679.1000398

Page 3 of 6

$$
\begin{aligned}
& V[i, j+1]=\frac{\Gamma[q j+1]}{\Gamma[q(\mathrm{j}+1)+1]}(-\alpha(i+1)(i+2)(i+3)) \mathrm{V}[i+3, \mathrm{j}] \\
& \mathrm{r}=0 \mathrm{~s}=0 \quad-3 \alpha \sum_{\gamma=0}^{i} \sum_{s=0}^{j}((i-\gamma+1)) U[\gamma, \mathrm{j}-\mathrm{s}] V[i-\gamma+1, \mathrm{~s}]
\end{aligned}
$$

The generalized differential transforms GDTM of the initial conditions can be obtained as follows:

\section{$\mathbf{U}(\mathbf{0}, 0)=\beta^{2} \operatorname{Sech}^{2}(0.5 \gamma)$}

$\mathrm{U}(1,0)=-\beta^{3} \operatorname{Sech}^{2}(0.5 \gamma) \operatorname{Tanh}(0.5 \gamma)$

$\mathrm{U}(2,0)=0.5 \beta^{2}\left(-0.5 \beta^{2} \operatorname{Sech}^{4}(0.5 \gamma)+\beta^{2} \operatorname{Sech}^{2}(0.5 \gamma) \operatorname{Tanh}^{2}(0.5 \gamma)\right)$

$\mathrm{U}(3,0)=0.1666666 \beta^{2}\left(2 \beta^{3} \operatorname{Sech}^{4}(0.5 \gamma) \operatorname{Tanh}(0.5 \gamma)-\beta^{3} \operatorname{Sech}^{2}\right.$ $\left.(0.5 \gamma) \operatorname{Tanh}^{3}(0.5 \gamma)\right)$

$\mathrm{U}(4,0)=.0416664 \beta^{2}\left(\beta^{4} \operatorname{Sech}^{6}(0.5 \gamma)-5.5 \beta^{4} \operatorname{Sech}^{4}(0.5 \gamma) \operatorname{Tanh}^{2}\right.$ $\left.(0.5 \gamma)+\beta^{4} \operatorname{Sech}^{2}(0.5 \gamma) \operatorname{Tanh}^{4}(0.5 \gamma)\right)$

$$
V(i, 0)=\sqrt{\frac{\alpha}{2}} U(i, 0)
$$

and so on.

we get the approximate solution as follows

$\mathrm{U}(\mathrm{x}, \mathrm{t})=0.196612+0.00567861 \mathrm{t}-0.0000689953 \mathrm{t}^{2}-5.02551^{\star} 10^{-6} \mathrm{t}^{3}-$ $3.24516^{\star} 10^{-8} \mathrm{t}^{4}+$

$2.25253^{\star} 10^{-9} t^{5}+4.66153^{\star} 10^{-11} t^{6}-4.66092^{\star} 10^{-13} t^{7}-2.76168^{\star} 10^{-14} t^{8}-$ $1.19556^{\star} 10^{-16} t^{9}-$

$0.0454289 \mathrm{x}+0.00110392 \mathrm{t} \mathrm{x}+0.000120612 \mathrm{t}^{2} \mathrm{x}+1.03845^{*} 10^{-6} \mathrm{t}^{3}$ $\mathrm{x}-9.01012^{\star} 10^{-8} \mathrm{t}^{4} \mathrm{x}-2.23753^{\star} 10^{-9} \mathrm{t}^{5} \mathrm{x}+2.61012^{\star} 10^{-11} \mathrm{t}^{6} \mathrm{x}+1.76461^{\star} 10^{-12}$ $\mathrm{t}^{7} \mathrm{x}+1.06761^{\star} 10^{-14} \mathrm{t}^{8} \mathrm{x}-1.26526^{\star} 10^{15} \mathrm{t}^{9} \mathrm{x}-0.0044157 \mathrm{x}^{2}-0.000964897 \mathrm{t} \mathrm{x^{2 } -}$ $0.0000124614 \mathrm{t}^{2} \mathrm{x}^{2}+1.44162^{\star} 10^{-6} \mathrm{t}^{3} \mathrm{x}^{2}+4.47507^{\star} 10^{-8} \mathrm{t}^{4} \mathrm{x}^{2}-6.2643^{\star} 10^{-10} \mathrm{t}^{5}$ $\mathrm{x}^{2}-4.94079^{\star} 10^{-11} \mathrm{t}^{6} \mathrm{x}^{2}-3.2944^{\star} 10^{-13} \mathrm{t}^{7} \mathrm{x}^{2}+2.85866^{\star} 10^{14} \mathrm{t}^{8} \mathrm{x}^{2}-1.62284^{\star} 10^{-16}$ $\mathrm{t}^{9} \mathrm{x}^{2}+0.00257306 \mathrm{x}^{3}+0.0000664609 \mathrm{t} \mathrm{x}^{3}-0.000011533 \mathrm{t}^{2} \mathrm{x}^{3}-4.77341^{\star} 10^{-7} \mathrm{t}^{3}$ $\mathrm{x}^{3}+8.35239^{\star} 10^{-9} \mathrm{t}^{4} \mathrm{x}^{3}+7.90527^{\star} 10^{-10} \mathrm{t}^{5} \mathrm{x}^{3}+6.14834^{\star} 10^{-12} \mathrm{t}^{6} \mathrm{x}^{3}-6.32837^{\star} 10^{-13}$ $\mathrm{t}^{7} \mathrm{x}^{3}-1.62358^{\star} 10^{-14} \mathrm{t}^{8} \mathrm{x}^{3}+1.03618^{\star} 10^{-15} \mathrm{t}^{9} \mathrm{x}^{3}-0.000132922 \mathrm{x}^{4}+0.0000461318$ $\mathrm{t} \mathrm{x}^{4}+2.86404^{\star} 10^{-6} \mathrm{t}^{2} \mathrm{x}^{4}-6.68191^{\star} 10^{-8} \mathrm{t}^{3} \mathrm{x}^{4}-7.90527^{\star} 10^{-9} \mathrm{t}^{4} \mathrm{x}^{4}-7.37801^{\star} 10^{-11} \mathrm{t}^{5}$ $\mathrm{x}^{4}+8.86132^{\star} 10^{-12} \mathrm{t}^{6} \mathrm{x}^{4}+2.57777^{\star} 10^{-13} \mathrm{t}^{7} \mathrm{x}^{4}-3.66086^{\star} 10^{-15} \mathrm{t}^{8} \mathrm{x}^{4}-1.33152^{\star} 10^{-16}$ $\mathrm{t}^{9} \mathrm{x}^{4}-0.0000738109 \mathrm{x}^{5}-9.16494^{\star} 10^{-6} \mathrm{t} \mathrm{x}^{5}+3.20732^{\star} 10^{-7} \mathrm{t}^{2} \mathrm{x}^{5}+5.05937^{\star} 10^{-8}$ $\mathrm{t}^{3} \mathrm{x}^{5}+5.9024^{\star} 10^{10} \mathrm{t}^{4} \mathrm{x}^{5}-8.5069^{\star} 10^{-11} \mathrm{t}^{5} \mathrm{x}^{5}-2.88991^{\star} 10^{-12} \mathrm{t}^{6} \mathrm{x}^{5}+5.21162^{\star} 10^{-14}$ $\mathrm{t}^{7} \mathrm{x}^{5}+4.66473^{\star} 10^{-15} \mathrm{t}^{8} \mathrm{x}^{5}-1.88001^{\star} 10^{-14} \mathrm{t}^{9} \mathrm{x}^{5}+0.0000122199 \mathrm{x}^{6}-8.55285^{\star} 10^{-7}$ $\mathrm{t} \mathrm{x}^{6}-2.02375^{\star} 10^{-7} \mathrm{t}^{2} \mathrm{x}^{6}-3.14795^{\star} 10^{-9} \mathrm{t}^{3} \mathrm{x}^{6}+5.67126^{\star} 10^{-10} \mathrm{t}^{4} \mathrm{x}^{6}+2.31194^{\star} 10^{-11}$ $\mathrm{t}^{5} \mathrm{x}^{6}-4.83771^{\star} 10^{-13} \mathrm{t}^{6} \mathrm{x}^{6}-4.71099^{\star} 10^{-14} \mathrm{t}^{7} \mathrm{x}^{6}-4.9457^{\star} 10^{-16} \mathrm{t}^{8} \mathrm{x}^{6}+2.12794^{\star} 10^{-14}$ $\mathrm{t}^{9} \mathrm{x}^{6}+9.77469^{\star} 10^{-7} \mathrm{x}^{7}+4.62571^{\star} 10^{-7} \mathrm{t} \mathrm{x} \mathrm{x}^{7}+1.0793^{\star} 10^{-8} \mathrm{t}^{2} \mathrm{x}^{7}-2.59258^{\star} 10^{-9} \mathrm{t}^{3}$ $\mathrm{x}^{7}-1.32111^{\star} 10^{-10} \mathrm{t}^{4} \mathrm{x}^{7}+3.3172^{\star} 10^{-12} \mathrm{t}^{5} \mathrm{x}^{7}+3.77056^{\star} 10^{-13} \mathrm{t}^{6} \mathrm{x}^{7}+3.29636^{\star} 10^{-15}$ $\mathrm{t}^{7} \mathrm{x}^{7}-5.21102^{\star} 10^{-16} \mathrm{t}^{8} \mathrm{x}^{7}+7.33238^{\star} 10^{-16} \mathrm{t}^{9} \mathrm{x}^{7}-4.62571^{\star} 10^{-7} \mathrm{x}^{8}-2.15859^{\star} 10^{8} \mathrm{t}$ $\mathrm{x}^{8}+7.77773^{\star} 10^{-9} \mathrm{t}^{2} \mathrm{x}^{8}+5.28443^{\star} 10^{-10} \mathrm{t}^{3} \mathrm{x}^{8}-1.6586^{\star} 10^{-11} \mathrm{t}^{4} \mathrm{x}^{8}-2.26224^{\star} 10^{-12} \mathrm{t}^{5}$ $\mathrm{x}^{8}-2.33268^{\star} 10^{-14} \mathrm{t}^{6} \mathrm{x}^{8}+4.02148^{\star} 10^{-15} \mathrm{t}^{7} \mathrm{x}^{8}+1.15006^{\star} 10^{-15} \mathrm{t}^{8} \mathrm{x}^{8}+3.5093^{\star} 10^{-14} \mathrm{t}^{9}$ $\mathrm{x}^{8}+1.91875^{\star} 10^{-8} \mathrm{x}^{9}-1.38271^{\star} 10^{-8} \mathrm{t} \mathrm{x}^{9}-1.40918^{\star} 10^{-9} \mathrm{t}^{2} \mathrm{x}^{9}+5.89724^{\star} 10^{-11} \mathrm{t}^{3} \mathrm{x}^{9}+$ $1.00544^{\star} 10^{11} \mathrm{t}^{4} \mathrm{x}^{9}+1.24348^{\star} 10^{-13} \mathrm{t}^{5} \mathrm{x}^{9}-2.50937^{\star} 10^{-14} \mathrm{t}^{6} \mathrm{x}^{9}-9.85678^{\star} 10^{-16} \mathrm{t}^{7} \mathrm{x}^{9}-$ $7.34662^{\star} 10^{-16} t^{8} x^{9}-2.55188^{\star} 10^{-14} t^{9} x^{9}$

$\mathrm{V}(\mathrm{x}, \mathrm{t})=0.098306+0.0028393 \mathrm{t}-0.0000344976 \mathrm{t}^{2}-2.51275^{\star} 10^{-6} \mathrm{t}^{3}-1.62258^{\star} 10^{-8}$ $t^{4}+1.12627^{\star} 10^{9} t^{5}+2.33076^{\star} 10^{-11} \quad t^{6}-2.33046^{\star} 10^{-13} t^{7}-1.38084^{\star} 10^{-14} t^{8}-5.979^{\star} 10$ $17 t^{9}-0.0227144 x+0.000551962 \mathrm{tx}+0.0000603061 \mathrm{t}^{2} \mathrm{x}+5.19226^{*} 10^{-7} \mathrm{t}^{3}$ $\mathrm{x}-4.50506^{\star} 10^{-8} \mathrm{t}^{4} \mathrm{x}-1.11877^{\star} 10^{-9} \mathrm{t}^{5} \mathrm{x}+1.30506^{\star} 10^{-11} \mathrm{t}^{6} \mathrm{x}+8.82307^{\star} 10^{-13}$ $t^{7} \mathrm{x}+5.33803^{\star} 10^{-15} \mathrm{t}^{8} \mathrm{x}-6.32564^{\star} 10^{-16} \mathrm{t}^{9} \mathrm{x}-0.00220785 \mathrm{x}^{2}-0.000482449 \mathrm{t}$ $\mathrm{x}^{2}-6.23071^{\star} 10^{-6} \mathrm{t}^{2} \mathrm{x}^{2}+7.2081^{\star} 10^{-7} \mathrm{t}^{3} \mathrm{x}^{2}+2.23753^{\star} 10^{-8} \mathrm{t}^{4} \mathrm{x}^{2}-3.13215^{\star} 10^{-10} \mathrm{t}^{5}$ $\mathrm{x}^{2}-2.4704^{\star} 10^{-11} \mathrm{t}^{6} \mathrm{x}^{2}-1.6472^{\star} 10^{-13} \mathrm{t}^{7} \mathrm{x}^{2}+1.42933^{\star} 10^{-14} \mathrm{t}^{8} \mathrm{x}^{2}-8.14247^{\star} 10^{-17} \mathrm{t}^{9}$

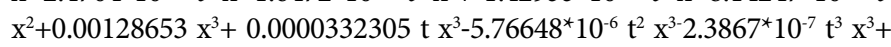
$4.1762^{\star} 10^{-9} \mathrm{t}^{4} \mathrm{x}^{3}+3.95263^{\star} 10^{-10} \mathrm{t}^{5} \mathrm{x}^{3}+3.07417^{\star} 10^{-12} \mathrm{t}^{6} \mathrm{x}^{3}-3.16418^{\star} 10^{-13} \mathrm{t}^{7} \mathrm{x}^{3}-$ $8.11788^{\star} 10^{-15} \mathrm{t}^{8} \mathrm{x}^{3}+5.18622^{\star} 10^{-16} \mathrm{t}^{9} \mathrm{x}^{3}-0.0000664609 \mathrm{x}^{4}+0.0000230659 \mathrm{t}$ $\mathrm{x}^{4}+1.43202^{\star} 10^{-6} \mathrm{t}^{2} \mathrm{x}^{4}-3.34096^{\star} 10^{-8} \mathrm{t}^{3} \mathrm{x}^{4}-3.95263^{\star} 10^{-9} \mathrm{t}^{4} \mathrm{x}^{4}-3.689^{\star} 10^{-11} \mathrm{t}^{5} \mathrm{x}^{4}+$ $4.43066^{\star} 10^{-12} \mathrm{t}^{6} \mathrm{x}^{4}+1.28889^{\star} 10^{-13} \mathrm{t}^{7} \mathrm{x}^{4}-1.83048^{\star} 10^{-15} \mathrm{t}^{8} \mathrm{x}^{4}-6.68102^{\star} 10^{-17}$ $\mathrm{t}^{9} \mathrm{x}^{4}-0.0000369055 \mathrm{x}^{5}-4.58247^{\star} 10^{-6} \mathrm{t} \mathrm{x}^{5}+1.60366^{\star} 10^{-7} \mathrm{t}^{2} \mathrm{x}^{5}+2.52969^{\star} 10^{-8} \mathrm{t}^{3}$ $\mathrm{x}^{5}+2.9512^{\star} 10^{-10} \mathrm{t}^{4} \mathrm{x}^{5}-4.25345^{\star} 10^{11} \mathrm{t}^{5} \mathrm{x}^{5}-1.44495^{\star} 10^{-12} \mathrm{t}^{6} \mathrm{x}^{5}+2.60581^{\star} 10^{-14} \mathrm{t}^{7}$ $\mathrm{x}^{5}+2.33245^{\star} 10^{-15} \mathrm{t}^{8} \mathrm{x}^{5}-9.39961^{\star} 10^{-15} \mathrm{t}^{9} \mathrm{x}^{5}+6.10996^{\star} 10^{-6} \mathrm{x}^{6}-4.27643^{\star} 10^{-7} \mathrm{t}$ $\mathrm{x}^{6}-1.01187^{\star} 10^{-7} \mathrm{t}^{2} \mathrm{x}^{6}-1.57397^{\star} 10^{-9} \mathrm{t}^{3} \mathrm{x}^{6}+2.83563^{\star} 10^{10} \mathrm{t}^{4} \mathrm{x}^{6}+1.15597^{\star} 10^{-11} \mathrm{t}^{5}$ $\mathrm{x}^{6}-2.41886^{\star} 10^{-13} \mathrm{t}^{6} \mathrm{x}^{6}-2.3555^{\star} 10^{-14} \mathrm{t}^{7} \mathrm{x}^{6}-2.47363^{\star} 10^{-16} \mathrm{t}^{8} \mathrm{x}^{6}+1.06401^{\star} 10^{-14}$ $\mathrm{t}^{9} \mathrm{x}^{6}+4.88734^{\star} 10^{-7} \mathrm{x}^{7+} 2.31286^{\star} 10^{-7} \mathrm{t} \mathrm{x}^{7}+5.39648^{\star} 10^{-9} \mathrm{t}^{2} \mathrm{x}^{7}-1.29629^{\star} 10^{9} \mathrm{t}^{3}$ $\mathrm{x}^{7}-6.60554^{\star} 10^{-11} \mathrm{t}^{4} \mathrm{x}^{7}+1.6586^{*} 10^{-12} \mathrm{t}^{5} \mathrm{x}^{7}+1.88528^{\star} 10^{-13} \mathrm{t}^{6} \mathrm{x}^{7}+1.64818^{\star} 10^{-15}$ $\mathrm{t}^{7} \mathrm{x}^{7}-2.60533^{\star} 10^{-16} \mathrm{t}^{8} \mathrm{x}^{7}+3.66782^{\star} 10^{-16} \mathrm{t}^{9} \mathrm{x}^{7}-2.31286^{\star} 10^{-7} \mathrm{x}^{8}-1.0793^{\star} 10^{-8} \mathrm{t}$ $\mathrm{x}^{8}+3.88887^{\star} 10^{-9} \mathrm{t}^{2} \mathrm{x}^{8}+2.64222^{\star} 10^{-10} \mathrm{t}^{3} \mathrm{x}^{8}-8.293^{\star} 10^{-12} \mathrm{t}^{4} \mathrm{x}^{8}-1.13112^{\star} 10^{-12} \mathrm{t}^{5}$ $x^{8}-1.16634^{\star} 10^{-14} t^{6} x^{8}+2.01073^{\star} 10^{-15} t^{7} x^{8}+5.75007^{\star} 10^{-16} t^{8} x^{8}+1.75465^{\star} 10^{-14}$ $\mathrm{t}^{9} \mathrm{x}^{8}+9.59375^{\star} 10^{-9} \mathrm{x}^{9}-6.91354^{\star} 10^{-9} \mathrm{t} \mathrm{x}^{9}-7.04591^{\star} 10^{-10} \mathrm{t}^{2} \mathrm{x}^{9}+2.94862^{*} 10^{-11}$ $\mathrm{t}^{3} \mathrm{x}^{9}+5.02719^{\star} 10^{-12} \mathrm{t}^{4} \mathrm{x}^{9}+6.21738^{\star} 10^{-14} \mathrm{t}^{5} \mathrm{x}^{9}-1.25468^{\star} 10^{-14} \mathrm{t}^{6} \mathrm{x}^{9}-4.92837^{\star} 10^{-16}$ $t^{7} x^{9}-3.67345^{\star} 10^{-16} t^{8} x^{9}-1.27594^{\star} 10^{-14} t^{9} x^{9}$

In Table 1 , the numerical values of the approximate and exact solutions for Example 1 show the accuracy and efficiency of our technique at different values of $\mathrm{x}, \mathrm{t}$. The absolute error is listed for different values of $x, t$. In Figure $1 a$, we consider fixed values $\alpha=\beta=0.5$, $\gamma=1$ and fixed order $q=1$ for piecewise approximation values of $x, t$ in the domain $-3 \leq \mathrm{x} \leq 3$ and $0.20 \leq \mathrm{t} \leq 1$.

In Figure $1 b$, we plot the exact solution with fixed values $\alpha=\beta=0.5$ and $\gamma=1$ in the domain $-3 \leq x$

$\leq 3$ and $0.20 \leq t \leq 1$. In Figure 2, we consider fixed values $\alpha=\beta=$ $0.5, Y=1$ and fixed order $q=0.5$ for piecewise approximation values of $\mathrm{x}$, $\mathrm{t}$ in the domain $-3 \leq \mathrm{x} \leq 3$ and $0.20 \leq \mathrm{t} \leq 1$.

\section{Example 2}

Consider the nonlinear dispersive long wave system of time fractional order [16-18], [19]

$$
D_{t}^{q 1} u=-v_{x}-\frac{1}{2}\left(u^{2}\right)_{x}, D_{t}^{q 2} v=-\left(u+u_{x x}+u v\right)_{x}
$$

\begin{tabular}{|c|c|c|c|c|c|c|c|c|c|}
\hline \multirow[t]{2}{*}{$\mathbf{x}$} & \multirow[t]{2}{*}{$\mathrm{t}$} & \multicolumn{2}{|c|}{$q=0.5$} & \multicolumn{2}{|c|}{$q=1$} & \multicolumn{2}{|c|}{$\alpha=\beta=0.5, y=1$} & \multicolumn{2}{|c|}{ Absolute Error } \\
\hline & & UGDTM & VGDTM & UGDTM & $V G D T M$ & $U E X$ & $V E X$ & $|U E X-U G D T M|$ & $|V E X-V G D T M|$ \\
\hline \multirow[t]{3}{*}{-3} & 0.2 & 0.25051 & 0.12526 & 0.24998 & 0.12499 & 0.24999 & 0.125 & 0.000013962 & $6.981 \times 10^{-6}$ \\
\hline & 0.4 & 0.26462 & 0.13231 & 0.24995 & 0.12497 & 0.24996 & 0.12498 & 0.000014232 & $7.116 \times 10^{-6}$ \\
\hline & 0.6 & 0.34368 & 0.17184 & 0.2499 & 0.12495 & 0.24991 & 0.12496 & 0.000014476 & $7.238 \times 10^{-6}$ \\
\hline \multirow[t]{3}{*}{0} & 0.2 & 0.19945 & 0.099723 & 0.19774 & 0.098872 & 0.19774 & 0.098872 & $5.551 \times 10^{-17}$ & $2.775 \times 10^{-17}$ \\
\hline & 0.4 & 0.20056 & 0.10028 & 0.19887 & 0.099436 & 0.19887 & 0.099436 & $5.551 \times 10^{-17}$ & $2.775 \times 10^{-17}$ \\
\hline & 0.6 & 0.20122 & 0.10061 & 0.19999 & 0.099997 & 0.19999 & 0.099997 & $8.327 \times 10^{-17}$ & $4.163 \times 10^{-17}$ \\
\hline \multirow[t]{3}{*}{2} & 0.2 & 0.10855 & 0.054273 & 0.15033 & 0.075166 & 0.15033 & 0.075166 & $9.8103 \times 10^{-9}$ & $4.9052 \times 10^{-9}$ \\
\hline & 0.4 & 0.13175 & 0.065877 & 0.15152 & 0.07576 & 0.15152 & 0.07576 & $1.0274 \times 10^{-8}$ & $5.137 \times 10^{-9}$ \\
\hline & 0.6 & 0.25378 & 0.12689 & 0.15271 & 0.076355 & 0.15271 & 0.076355 & $1.0729 \times 10^{-8}$ & $5.3644 \times 10^{-9}$ \\
\hline
\end{tabular}

For $0<\mathrm{q} 1, \mathrm{q} 1<1$, with initial condition given by

Table 1: Numerical values when $q=0.5,1$ and $\alpha=\beta=0.5, Y=1$ for Example 1. 


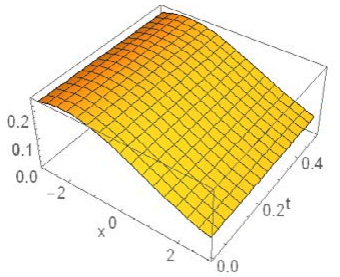

$\mathrm{U}(\mathrm{x}, \mathrm{t})$

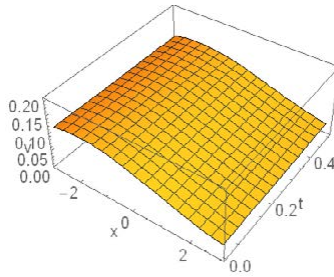

$\mathrm{v}(\mathrm{x}, \mathrm{t})$
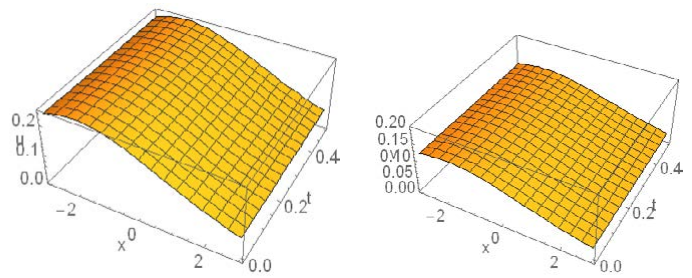

(b)

Figure 1: (a) The graph for the approximate solution of Example 1 for $\alpha=\beta=0.5, Y=1$ and $q=1$ (b) The graph for the exact solution of Example 1 for $\alpha$ $=\beta=0.5$ and $Y=1$.

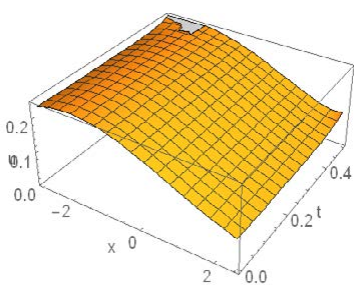

$\mathrm{U}(\mathrm{x}, \mathrm{t})$

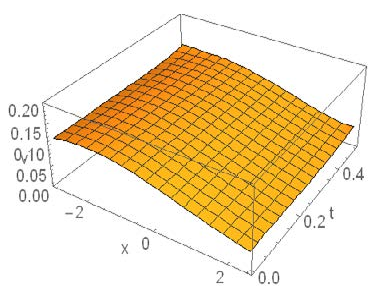

$\mathrm{v}(\mathrm{x}, \mathrm{t})$

Figure 2: The graph for the approximate solution of Example 1 for $\alpha=\beta=0.5, Y=1$ and $q=0.5$.

$$
u(x, 0)=\alpha\left[\tanh \left(\frac{1}{2}\left[\beta+\alpha x-\alpha^{2} t\right]+1\right)\right], v(x, 0)=-1+\frac{1}{2} \alpha^{2} \sec h^{2}\left(\frac{1}{2}[\beta+\alpha x]\right)
$$

For $\mathrm{q} 1=\mathrm{q} 2=1$, the system $(43)$ has the following exact solitary wave solutions:

$$
\begin{aligned}
& u(x, 0)=\alpha\left[\tanh \left(\frac{1}{2}\left[\beta+\alpha x-\alpha^{2} t\right]+1\right)\right] \\
& v(x, 0)=-1+\frac{1}{2} \alpha^{2} \sec h^{2}\left(\frac{1}{2}\left[\beta+\alpha x-\alpha^{2} t\right]\right)
\end{aligned}
$$

Where $\beta, \alpha$ are arbitrary constants

Applying the generalized differential transform GDTM to both sides of (8), and by using the related theorems, we will have

$$
\begin{aligned}
U[i, \mathrm{j}+1]= & \frac{\Gamma[q j+1]}{\Gamma[q(\mathrm{j}+1)+1]}\left(\begin{array}{l}
-(i+1) V[i+1, j] \\
-\frac{1}{2} \sum_{\gamma=0}^{i} \sum_{s=0}^{j}((i-\gamma+1)) U[\gamma, \mathrm{j}-\mathrm{s}] U[i-\gamma+1, \mathrm{~s}]
\end{array}\right. \\
V[i, j+1]= & \frac{\Gamma[q 2 j+1]}{\Gamma[q 2(\mathrm{j}+1)+1]}(-(i+1) U[i+1, \mathrm{j}])-(i+1)(i+2)(i+3) U[i+3, j] \\
& -\sum_{\gamma=0}^{i} \sum_{s=0}^{j}((\gamma+1)) U[\gamma+1, \mathrm{j}-\mathrm{s}] V[i-\gamma, s] \\
& \left.\left.-\sum_{\gamma=0}^{i} \sum_{s=0}^{j}((\gamma+1)) U[\gamma+1, \mathrm{j}-\mathrm{s}] U[i-\gamma, s]\right]\right]
\end{aligned}
$$

The Generalized differential transforms GDTM of the initial conditions can be obtained as follows:

$$
\begin{aligned}
& \mathrm{U}(0,0)=\alpha(1+\operatorname{Tanh}(0.5 \beta)) \\
& \mathrm{U}(1,0)=0.5 \alpha^{2} \operatorname{Sech}^{2}(0.5 \beta) \\
& \mathrm{U}(2,0)=-0.25 \alpha^{3} \operatorname{Sech}^{2}(0.5 \beta) \operatorname{Tanh}(0.5 \beta)
\end{aligned}
$$

$\mathrm{U}(3,0)=0.166667 \alpha\left(-0.25 \alpha^{3} \operatorname{Sech}^{4}(0.5 \beta)+0.5 \alpha^{3} \operatorname{Sech}^{2}(0.5 \beta)\right.$ $\left.\operatorname{Tanh}^{2}(0.5 \beta)\right)$

$\mathrm{U}(4,0)=0.0416667 \alpha\left(\alpha^{4} \operatorname{Sech}^{4}(0.5 \beta) \operatorname{Tanh}(0.5 \beta)-0.5 \alpha^{4} \operatorname{Sech}^{2}(0.5\right.$ B) $\left.\operatorname{Tanh}^{3}(0.5 \beta)\right) \mathrm{V}(0,0)=-1+0.5 \alpha^{2} \operatorname{Sech}^{2}(0.5 \beta)$

$\mathrm{V}(1,0)=-0.5 \alpha^{3} \operatorname{Sech}^{2}(0.5 \beta) \operatorname{Tanh}(0.5 \beta)(13)$

$\mathrm{V}(2,0)=0.25 \alpha^{2}\left(-0.5 \alpha^{2} \operatorname{Sech}^{4}(0.5 \beta)+\alpha^{2} \operatorname{Sech}^{2}(0.5 \beta) \operatorname{Tanh}^{2}(0.5 \beta)\right)$

$\mathrm{V}(3,0)=0.0833333 \alpha^{2}\left(2 . \alpha^{3} \operatorname{Sech}^{4}(0.5 \beta) \operatorname{Tanh}(0.5 \beta)-\alpha^{3} \operatorname{Sech}^{2}(0.5\right.$ ק) $\operatorname{Tanh}^{3}(0.5 \beta)$

$\mathrm{V}(4,0)=0.0208333 \alpha^{2}\left(\alpha^{4} \operatorname{Sech}^{6}(0.5 \quad \beta)-5.5 \alpha^{4} \operatorname{Sech}^{4}(0.5 \quad \beta)\right.$ $\operatorname{Tanh}^{2}(0.5 \beta)+\alpha^{4}$

$\left.\operatorname{Sech}^{2}(0.5 \beta) \operatorname{Tanh}^{4}(0.5 \beta)\right)$

and so on.

we get the approximate solution as follows

$\mathbf{U}(\mathbf{x}, \mathbf{t})=0.622459 \quad-0.0221809 \quad \mathrm{t}-0.00385653 \quad \mathrm{t}^{2}+0.000207839 \quad \mathrm{t}^{3}-$ 
Citation: Kawala AM (2018) Numerical Technique for a System of Nonlinear Partial Differential Equations of the Time-Fractional Order. J Appl Computat Math 7: 398. doi: 10.4172/2168-9679.1000398

Page 5 of 6

$0.0000345697 \quad t^{4}-2.06478^{\star} 10^{-6} \quad t^{5}+0.0000196709 \quad t^{6}-0.0000183349$ $\mathrm{t}^{7}+0.0000239926 \quad \mathrm{t}^{8}-0.000021996 \quad \mathrm{t}^{9}+0.117502 \quad \mathrm{x}+0.0096196 \quad \mathrm{t}$ $\begin{array}{lllllll}\mathrm{x}+0.0010327 \quad \mathrm{t}^{2} & \mathrm{x}-0.000422623 \quad \mathrm{t}^{3} & \mathrm{x}+0.000292331 \mathrm{t}^{4} & \mathrm{x}-0.000137416\end{array}$ $\begin{array}{lllllll}t^{5} & x+0.000104533 & t^{6} & x-0.0000629452 & t^{7} & x+0.0000410731 & t^{8}\end{array}$ $\mathrm{x}-0.0000204109 \mathrm{t}^{9} \mathrm{x}-0.0071946 \mathrm{x}^{2} 0.000131237 \mathrm{t} \mathrm{x}^{2}+0.000852079 \mathrm{t}^{2} \mathrm{x}^{2}-$ $0.000202683 \mathrm{t}^{3} \mathrm{x}^{2}+0.000033885 \mathrm{t}^{4} \mathrm{x}^{2}+1.86867^{\star} 10^{-6} \mathrm{t}^{5} \mathrm{x}^{2}-0.0000579999 \mathrm{t}^{6}$ $\mathrm{x}^{2}+0.0000656083 \mathrm{t}^{7} \mathrm{x}^{2}-0.000101972 \mathrm{t}^{8} \mathrm{x}^{2}+0.000113379 \mathrm{t}^{9} \mathrm{x}^{2} 0.00200743$

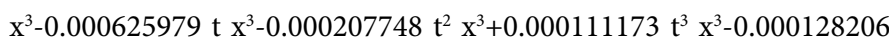

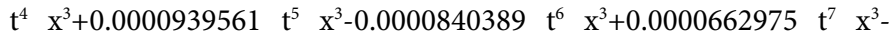
$0.000040982 t^{8} x^{3}+0.0000153392 t^{9} x^{3}+0.000272802 x^{4}+0.0000829088$ t $\mathrm{x}^{4}-0.0000614468 \mathrm{t}^{2} \quad \mathrm{x}^{4}+0.0000377461 \quad \mathrm{t}^{3} \quad \mathrm{x}^{4}-1.17137^{\star} 10^{-6} \quad \mathrm{t}^{4} \quad \mathrm{x}^{4}-$ $6.66086^{\star} 10^{-6} \mathrm{t}^{5} \mathrm{x}^{4}+0.0000371152 \mathrm{t}^{6} \mathrm{x}^{4} 0.0000504916 \mathrm{t}^{7} \mathrm{x}^{4}+0.0000865814$ $t^{8} x^{4}-0.000114065 t^{9} x^{4}+0.0000353178 x^{5}+0.0000222 t x^{5}+0.000019124$ $\begin{array}{lllllll}t^{2} & x^{5}-0.0000167275 & t^{3} & x^{5}+0.0000236157 & t^{4} & x^{5}-0.0000247831 & t^{5}\end{array}$ $\mathrm{x}^{5}+0.0000243714 \quad \mathrm{t}^{6} \quad \mathrm{x}^{5}-0.0000234354 \quad \mathrm{t}^{7} \quad \mathrm{x}^{5}+9.8599^{\star} 10^{-6} \quad \mathrm{t}^{8}$ $\mathrm{x}^{5}+6.83437^{\star} 10^{-6} \mathrm{t}^{9} \mathrm{x}^{5}-8.4704^{\star} 10^{-6} \mathrm{x}^{6}-5.69213^{*} 10^{-6} \mathrm{t} \mathrm{x}^{6}+2.63208^{\star} 10^{-6} \mathrm{t}^{2}$ $\mathrm{x}^{6}-3.41567^{\star} 10^{-6} \mathrm{t}^{3} \mathrm{x}^{6}-1.37309^{\star} 10^{-6} \mathrm{t}^{4} \mathrm{x}^{6}+3.21496^{\star} 10^{-6} \mathrm{t}^{5} \mathrm{x}^{6}-0.0000114965$ $\mathrm{t}^{6} \quad \mathrm{x}^{6}+0.0000186472 \quad \mathrm{t}^{7} \quad \mathrm{x}^{6}-0.0000342155 \quad \mathrm{t}^{8} \quad \mathrm{x}^{6}+0.0000521823 \quad \mathrm{t}^{9}$ $\mathrm{x}^{6} 4.52116^{\star} 10^{-7} \mathrm{x}^{7}-4.53159^{\star} 10^{-7} \mathrm{t} \mathrm{x}^{7}-1.21818^{\star} 10^{-6} \mathrm{t}^{2} \mathrm{x}^{7}+1.75717^{\star} 10^{-6} \mathrm{t}^{3}$ $\mathrm{x}^{7}-2.70689^{\star} 10^{-6} \mathrm{t}^{4} \mathrm{x}^{7}+3.8071^{\star} 10^{-6} \mathrm{t}^{5} \mathrm{x}^{7}-3.81954^{\star} 10^{-6} \mathrm{t}^{6} \mathrm{x}^{7}+4.04573^{\star} 10^{-6}$ $\mathrm{t}^{7} \mathrm{x}^{7}+9.71233^{\star} 10^{-7} \mathrm{t}^{8} \mathrm{x}^{7}-9.0206^{\star} 10^{-6} \mathrm{t}^{9} \mathrm{x}^{7}+2.31548^{\star} 10^{-7} \mathrm{x}^{8}+2.57413^{\star} 10^{-7} \mathrm{t}$ $\mathrm{x}^{8}-6.46656^{\star} 10^{-8} \mathrm{t}^{2} \mathrm{x}^{8}+1.75893^{\star} 10^{-7} \mathrm{t}^{3} \mathrm{x}^{8}+3.17841^{\star} 10^{-7} \mathrm{t}^{4} \mathrm{x}^{8}-7.32868^{\star} 10^{-7} \mathrm{t}^{5}$ $x^{8}+2.23131^{\star} 10^{-6} t^{6} x^{8}-4.25847^{\star} 10^{-6} t^{7} x^{8}+8.21701^{\star} 10^{-6} t^{8} x^{8}-0.0000141522$ $\mathrm{t}^{9} \mathrm{x}^{8}-2.76026^{\star} 10^{-10} \mathrm{x}^{9}-1.61687^{\star} 10^{-9} \mathrm{t} \mathrm{x}^{9}+6.16295^{\star} 10^{-8} \mathrm{t}^{2} \mathrm{x}^{9}-1.3936^{\star} 10^{-7} \mathrm{t}^{3}$ $x^{9}+2.2317^{\star} 10^{-7} t^{4} x^{9}-3.97295^{\star} 10^{-7} t^{5} x^{9}+3.56999^{\star} 10^{-7} t^{6} x^{9}-3.13469^{\star} 10^{-7} t^{7}$ $x^{9}-1.04624^{\star} 10^{-6} t^{8} x^{9}+3.80389^{*} 10^{-6} t^{9} x^{9}$.

$\mathrm{V}(\mathbf{x}, \mathbf{t})=-0.882498+0.0071946$

$\mathrm{t}-0.00114381$ $t^{3}-0.000211192 \quad t^{4}+0.00016243 \quad t^{5} 0.0000871647$ $t^{7}-0.0000363404 \quad t^{8}+0.0000142794 \quad t^{9}-0.0143892 \quad x+0.0060223$ $\mathrm{x}-0.000611667 \mathrm{t}^{2} \mathrm{x}+0.000287603 \mathrm{t}^{3} \mathrm{x}-0.0000823225 \mathrm{t}^{4} \mathrm{x}-0.0000726499$ $t^{5} x+0.0000937663 t^{6} x-0.00017051 t^{7} x+0.000183372 t^{8} x-0.000250121$ $\mathrm{t}^{9} \mathrm{x}-0.0060223 \mathrm{x}^{2} 0.00163681 \mathrm{t} \quad \mathrm{x}^{2}+0.000269607 \mathrm{t}^{2} \quad \mathrm{x}^{2}-0.000491773$ $\begin{array}{llllllll}t^{3} & x^{2}+0.00031687 & t^{4} & x^{2}-0.000307409 & t^{5} & x^{2}+0.000232876 & t^{6} & x^{2}-\end{array}$
$0.000182094 t^{7} x^{2}+0.000122064 t^{8} x^{2}-6.78186^{\star} 10^{-7} t^{9} x^{2}+0.00109121 x^{3}-$ $0.000353178 \mathrm{t} \mathrm{x}^{3}+0.000210813 \mathrm{t}^{2} \mathrm{x}^{3}-0.0000767257 \mathrm{t}^{3} \mathrm{x}^{3}+0.0000372177$ $t^{4} \quad x^{3}+0.0000850959 \quad t^{5} \quad x^{3}-0.000122551 \quad t^{6} \quad x^{3}+0.000246843 \quad t^{7} \quad x^{3}-$ $0.000321015 \mathrm{t}^{8} \mathrm{x}^{3}+0.000484615 \mathrm{t}^{9} \mathrm{x}^{3}+0.000176589 \mathrm{x}^{4}+0.000127056 \mathrm{t} \mathrm{x}-$ $0.0000434962 t^{2} x^{4}+0.000109602 t^{3} x^{4}-0.000109591 t^{4} x^{4}+0.000123244$

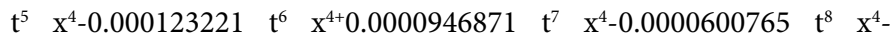
$0.0000893586 \mathrm{t}^{9} \quad \mathrm{x}^{4}-0.0000508224 \quad \mathrm{x}^{5}+9.49444^{*} 10^{-6} \quad \mathrm{t} \quad \mathrm{x}^{5} 0.000025148$ $t^{2} \quad x^{5}+4.93675^{\star} 10^{-6} \quad t^{3} \quad x^{5}-1.33756^{\star} 10^{-6} \quad t^{4} \quad x^{5}-0.0000359522 \quad t^{5} \quad x^{5}$ $+0.0000604517 \mathrm{t}^{6} \mathrm{x}^{5}-0.000128005 \mathrm{t}^{7} \mathrm{x}^{5}+0.000196992 \mathrm{t}^{8} \mathrm{x}^{5}-0.000319158$ $\mathrm{t}^{9} \mathrm{x}^{5}-3.16481^{\star} 10^{-6} \mathrm{x}^{6}-6.48334^{\star} 10{ }^{6}{ }^{6} \mathrm{x}^{6}+5.57826^{\star} 10^{-6} \mathrm{t}^{2} \mathrm{x}^{6}-0.0000136042$ $t^{3} \quad x^{6}+0.0000196537 \quad t^{4} \quad x^{6}-0.0000240221 \quad t^{5} \quad x^{6}+0.0000296963 \quad t^{6} \quad x^{6}-$ $0.0000179167 \mathrm{t}^{7} \mathrm{x}^{6}+3.54205^{\star} 10^{-7} \mathrm{t}^{8} \mathrm{x}^{6}+0.0000911456 \mathrm{t}^{9} \mathrm{x}^{6}+1.85238^{\star} 10^{-6}$ $\mathrm{x}^{7}+9.93692^{\star} 10^{-9} \mathrm{t} \mathrm{x}^{7}+1.76202^{\star} 10^{-6} \mathrm{t}^{2} \mathrm{x}^{7}+3.5987^{\star} 10^{-7} \mathrm{t}^{3} \mathrm{x}^{7}-1.24649^{\star} 10$ ${ }^{6} \mathrm{t}^{4} \mathrm{x}^{7}+8.37683^{*} 10^{-6} \quad \mathrm{t}^{5} \quad \mathrm{x}^{7}-0.0000166504 \quad \mathrm{t}^{6} \quad \mathrm{x}^{7}+0.0000366581 \mathrm{t}^{7} \mathrm{x}^{7}-$ $0.0000653338 \mathrm{t}^{8} \mathrm{x}^{7}+0.000110358 \mathrm{t}^{9} \mathrm{x}^{7}-2.48423^{*} 10^{-9} \mathrm{x}^{8}+2.56792^{\star} 10^{-7} \mathrm{t}^{8}-$ $5.12351^{\star} 10^{-7} \mathrm{t}^{2} \mathrm{x}^{8}+1.17761^{\star} 10^{-6} \mathrm{t}^{3} \mathrm{x}^{8}-2.31296^{\star} 10^{-6} \mathrm{t}^{4} \mathrm{x}^{8}+2.87974^{\star} 10^{-6} \mathrm{t}^{5}$ $\mathrm{x}^{8}-4.05858^{\star} 10^{-6} \mathrm{t}^{6} \mathrm{x}^{8}+2.03405^{\star} 10^{-7} \mathrm{t}^{7} \mathrm{x}^{8}+7.35912^{\star} 10^{-6} \mathrm{t}^{8} \mathrm{x}^{8}-0.0000421974$ $\mathrm{t}^{9} \mathrm{x}^{8}-5.70648^{\star} 10^{-8} \mathrm{x}^{9}-1.58831^{\star} 10^{-8} \mathrm{tx}^{9}-8.20714^{\star} 10^{-8} \mathrm{t}^{2} \mathrm{x}^{9}-1.02148^{\star} 10^{-7} \mathrm{t}^{3}$ $x^{9}+3.02919^{*} 10^{-7} t^{4} x^{9}-1.30365^{\star} 10^{-6} t^{5} x^{9}+3.04502^{\star} 10^{-6} t^{6} x^{9}-6.93429^{*} 10^{-6}$ $t^{7} x^{9}+0.0000139871 t^{8} x^{9}-0.0000236784 t^{9} x^{9}$.

In Table 2, the numerical values of the approximate and exact solutions for Example 2 show the accuracy and efficiency of our technique at different values of $\mathrm{x}, \mathrm{t}$. The absolute error is listed for different values of $x$, t. In Figure 3a, we consider fixed values $\alpha=\beta=0.5$ and fixed order $\mathrm{q} 1=\mathrm{q} 2=1$ for piecewise approximation values of $\mathrm{x}, \mathrm{t}$ in the domain $-3 \leq \mathrm{x} \leq 3$ and $0.20 \leq \mathrm{t} \leq 1$.

In Figure 3b, we plot the exact solution with fixed values $\alpha=\beta=0.5$ in the domain $-3 \leq x \leq 3$ and $0.20 \leq t \leq 1$. In Figure 4 , we consider fixed values $\alpha=\beta=0.5$ and fixed order $\mathrm{q} 1=\mathrm{q} 2=0.5$ for piecewise approximation values of $x, t$ in the domain $-3 \leq x \leq 3$ and $0.20 \leq t \leq 1$.

\section{Conclusions}

In this paper, a nonlinear wave equations systems of time-fractional

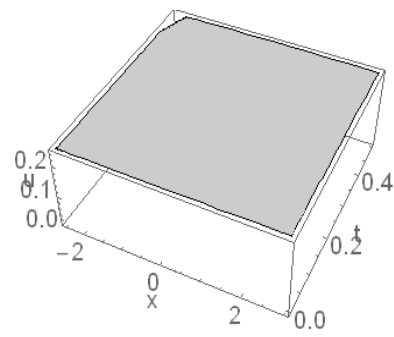

$\mathrm{U}(\mathrm{x}, \mathrm{t})$

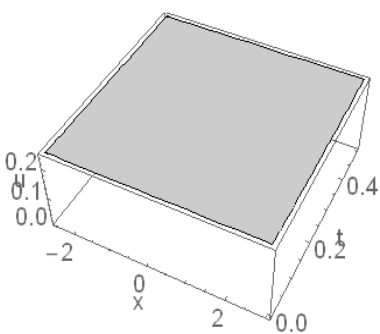

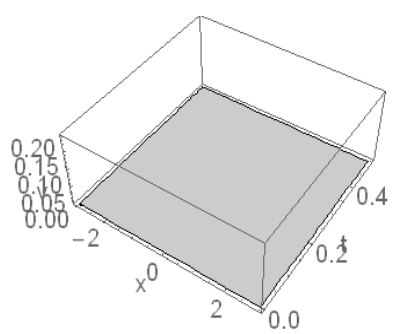

(a)

$\mathrm{v}(\mathrm{x}, \mathrm{t})$

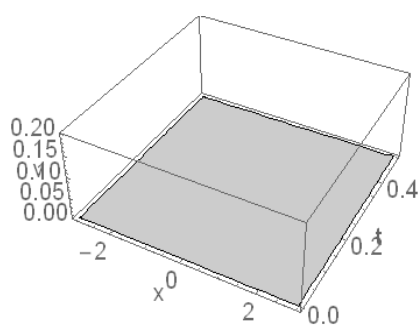

(b)

Figure 3: (a) The graph for the approximate solution of Example 2 for $\alpha=\beta=0.5$ and $q 1=q 2=1$ (b) The graph for the exact solution of Example 2 for $\alpha=\beta=0.5$. 
Citation: Kawala AM (2018) Numerical Technique for a System of Nonlinear Partial Differential Equations of the Time-Fractional Order. J Appl Computat Math 7: 398. doi: 10.4172/2168-9679.1000398

Page 6 of 6

\begin{tabular}{|c|c|c|c|c|c|c|c|c|c|}
\hline \multirow[t]{2}{*}{$\mathbf{x}$} & \multirow[t]{2}{*}{$\mathbf{t}$} & \multicolumn{2}{|c|}{$q 1=q 2=0.5$} & \multicolumn{2}{|l|}{$q 1=q 2=1$} & \multicolumn{2}{|l|}{$\alpha=\beta=0.5$} & \multicolumn{2}{|l|}{ Absolute Error } \\
\hline & & UGDTM & $V G D T M$ & $U G D T M$ & VGDTM & UEX & $V E X$ & $|U E X-U G D T M|$ & $|V E X-V G D T M|$ \\
\hline \multirow[t]{3}{*}{-3} & 0.2 & 0.34715 & -0.89709 & 0.24998 & 0.12499 & 0.24999 & 0.125 & 0.000013962 & $6.9812 \times 10^{-6}$ \\
\hline & 0.4 & 0.080769 & -1.1115 & 0.24995 & 0.12497 & 0.24996 & 0.12498 & 0.000014232 & $7.1159 \times 10^{-6}$ \\
\hline & 0.6 & -1.375 & -2.2218 & 0.2499 & 0.12497 & 0.24991 & 0.12496 & 0.000014476 & $7.238 \times 10^{-6}$ \\
\hline \multirow[t]{3}{*}{0} & 0.2 & 0.60972 & 0.87911 & 0.19774 & 0.098872 & 0.19774 & 0.098872 & $5.551 \times 10^{-17}$ & $2.776 \times 10^{-17}$ \\
\hline & 0.4 & 0.60203 & 0.87682 & 0.19887 & 0.099436 & 0.19887 & 0.099436 & $5.551 \times 10^{-17}$ & $2.776 \times 10^{-17}$ \\
\hline & 0.6 & 0.58754 & 0.87031 & 0.19999 & 0.099997 & 0.19999 & 0.099997 & $8.326 \times 10^{-17}$ & $4.163 \times 10^{-17}$ \\
\hline \multirow[t]{3}{*}{2} & 0.2 & 0.72348 & -0.89645 & 0.15033 & 0.075166 & 0.15033 & 0.075166 & $9.8103 \times 10^{-9}$ & $4.9052 \times 10^{-9}$ \\
\hline & 0.4 & 0.72077 & -0.89622 & 0.15152 & 0.07576 & 0.15152 & 0.07576 & $1.0274 \times 10^{-8}$ & $5.137 \times 10^{-9}$ \\
\hline & 0.6 & 0.72345 & -0.9051 & 0.15271 & 0.076355 & 0.15271 & 0.076355 & $1.0729 \times 10^{-8}$ & $5.3644 \times 10^{-9}$ \\
\hline
\end{tabular}

Table 2: Numerical values when $q 1=q 2=0.5,1$ and $\alpha=\beta=0.5$ for Example 2.

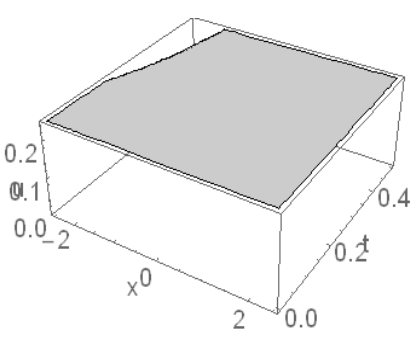

$\mathrm{U}(\mathrm{x}, \mathrm{t})$

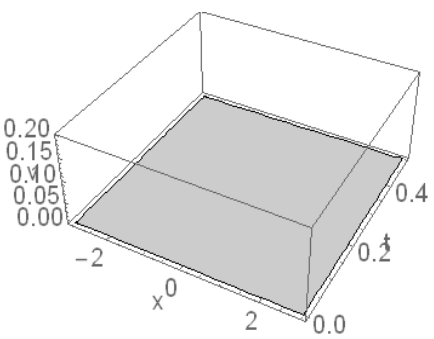

$\mathrm{V}(\mathrm{x}, \mathrm{t})$

Figure 4: The graph for the approximate solution of Example 2 for $\alpha=\beta=0.5$ and $q 1=q 2=0.5$.

order via generalized Differential transform method (GDTM) was studied. From the computational point of view, the solutions obtained by our technique were in excellent agreement with the exact solution to confirm the effectiveness and accuracy. We used Mathematica software to obtain the approximate and numerical results as well as drawing the graphs.

\section{References}

1. Zhou JK (1986) Differential transformation and its applications for electrical circuits.

2. Ayaz F (2003) On the two-dimensional differential transform method. Appl Math Comput 143: 361- 374.

3. Ayaz F (2004) Solutions of the system of differential equations by differential transform method. Appl Math Comput 147: 547-567.

4. Chen CK, Ho SH (1996) Application of differential transformation to eigenvalue problems. Appl Math Comput 79: 173-188.

5. Kurnaz A, Oturanc G, Kiris ME (2005) n-Dimensional differential transformation method for solving PDEs. Int J Comput Math 82: 369-380.

6. Podlubny I (1999) Fractional-order systems and PI/sup/spl lambda//D/sup/spl mu//-controllers. IEEE Transactions on automatic control 44: 208-214.

7. Zhou JK (1986) Differential Transformation and Its Applications for Electronic Circuits, Huazhong Science \& Technology University Press, China.

8. Bildik N, Konuralp A, Bek FO, Küçükarslan S (2006) Solution of different type of the partial differential equation by differential transform method and Adomian's decomposition method. Appl Math Comput 172: 551-567.

9. Hassan IAH (2008) Comparison differential transformation technique with Adomian decomposition method for linear and nonlinear initial value problems. Chaos, Solitons \& Fractals 36: 53-65.
10. Odibat ZM, Shawagfeh NT (2007) Generalized Taylor's formula. Appl Math Comput 186: 286-293.

11. Caputo $M$ (1967) Linear models of dissipation whose $Q$ is almost frequency independent-II. Geophysical Journal International 13: 529-539.

12. Liu J, Hou G (2011) Numerical solutions of the space-and time-fractional coupled Burgers equations by generalized differential transform method. Appl Math Comput 217: 7001-7008.

13. Podlubny I (1998) Fractional differential equations: an introduction to fractiona derivatives, fractional differential equations, to methods of their solution and some of their applications. Elsevier 198

14. Jafari H, Seifi $S$ (2009) Solving a system of nonlinear fractional partial differential equations using homotopy analysis method. Commun Nonlinear Sci Numer Simul 14: 1962-1969.

15. Momani S, Odibat Z (2008) A novel method for nonlinear fractional partial differential equations: Combination of DTM and generalized Taylor's formula. J Comput Appl Math 220: 85-95.

16. Wang M, Zhou Y, Li Z (1996) Application of a homogeneous balance method to exact solutions of nonlinear equations in mathematical physics. Physics Letters A 216: $67-75$

17. Lou SY (1993) Painlevé test for the integrable dispersive long wave equations in two space dimensions. Physics Letters A 176: 96-100.

18. Paquin G, Winternitz P (1990) Group theoretical analysis of dispersive long wave equations in two space dimensions. Physica D: Nonlinear Phenomena 46: 122-138.

19. Hirota R, Satsuma J (1981) Soliton solutions of a coupled Korteweg-de Vries equation. Physics Letters A 85: 407-408. 